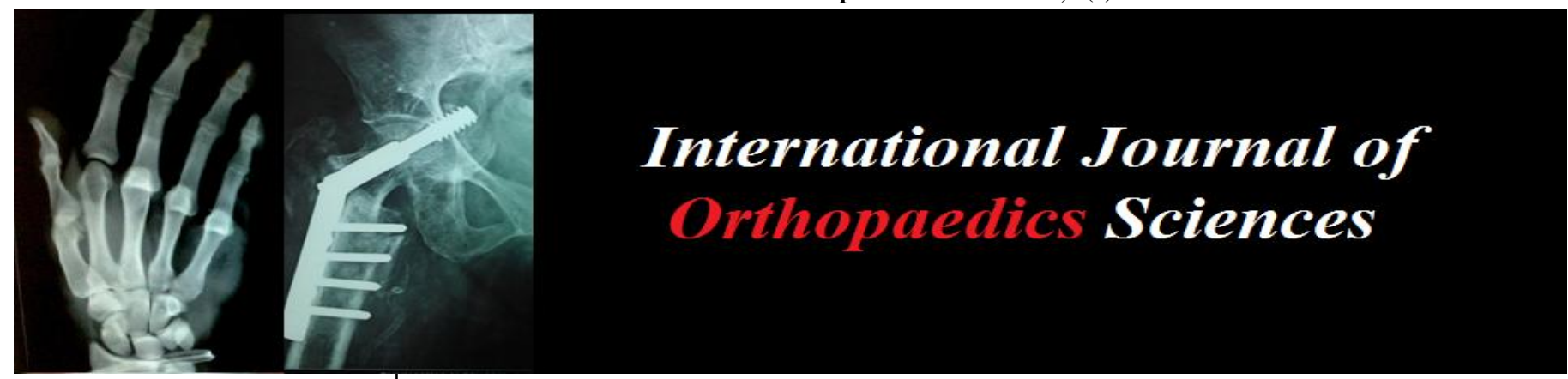

ISSN: $2395-1958$

IJOS 2017; 3(4): 394-399

(C) 2017 IJOS

www.orthopaper.com

Received: 05-05-2017

Accepted: 08-06-2017

Dr. Ankit Patel

Orthopeadic Department, Dhiraj Hospital, Sbks Mirc, Pipariya,

Vadodara, Gujurat, India

Dr. Parth Thakor

Orthopeadic Department, Dhiraj

Hospital, Sbks Mirc, Pipariya,

Vadodara, Gujurat, India

Dr. Jainish Patel

Orthopeadic Department, Dhira

Hospital, Sbks Mirc, Pipariya,

Vadodara, Gujurat, India

Dr. Dhruven Kosada

Orthopeadic Department, Dhira

Hospital, Sbks Mirc, Pipariya,

Vadodara, Gujurat, India

Dr. Sarvang Desai

Orthopeadic Department, Dhiraj

Hospital, Sbks Mirc, Pipariya,

Vadodara, Gujurat, India
Correspondence

Dr. Jainish Patel

Orthopeadic Department, Dhiraj

Hospital, Sbks Mirc, Pipariya,

Vadodara, Gujurat, India

\section{Retrospective critical analysis of treatment of closed extra capsular proximal Femur fractures with proximal femur nail at rural teaching setup}

\author{
Dr. Ankit Patel, Dr. Parth Thakor, Dr. Jainish Patel, Dr. Dhruven Kosada \\ and Dr. Sarvang Desai
}

DOI: https://doi.org/10.22271/ortho.2017.v3.i4f.53

\section{Abstract}

Background: For extracapsular proximal femur fractures, the treatment options are innumerous, though the implant choice is debatable. In our institute we use proximal femoral nail for the treatment of extracapsular proximal femur fractures. Choosing most suitable implant for such fractures specially in old aged osteoporotic patients till date puts the surgeons in great dilemma.

Aim \& Objective: To study the clinical results and functional outcome of proximal femoral nail in extracapsular proximal femur fractures routinely used in our institute and to review the available literature.

Material \& Methods: This study consists of 60 patients who had undergone treatment of proximal femur fractures. Males constituted $70 \%$ of the study and $43.6 \%$ of the patients were more than 60 years old. Clinical outcome and functional results were evaluated by using Harris Hip scoring system.

Results: Mean duration of surgery was 93 min.Post-operative complication rate was $6.7 \%$ which was superficial infection. Average time of union is 11.7 weeks. One patient had shortening of $2 \mathrm{cms}$ and 2 patients had shortening of $1 \mathrm{~cm}$. According to the Harris Hip Score we got excellent result in $70 \%$ and good result in $20 \%$.

Conclusion: Proximal femur fracture is common in elderly due to osteoporosis and mostly occurs due to trivial fall. PFN being a closed procedure, the amount of blood loss and duration of surgery was lesser. PFN is technically easier, with least complications even on follow up. PFN achieved the best clinical results and highest functional scores. Hence we conclude that PFN is the treatment of choice in proximal femur fracture.

Keywords: retrospective critical analysis, treatment, rural teaching setup

\section{Introduction}

Fractures of the proximal third femur and hip are relatively common injuries in adults. These fractures are associated with substantial morbidity and mortality; $30 \%$ of elderly patients die

Within 1 year of fracture. After 1 year, patients seem to resume their age - adjusted mortality rate.

Trochanteric fractures are common in the elderly people. The frequency of these fractures has increased due to, the increasing life span and more sedentary lifestyle brought on by urbanization. Trochanteric fractures occur in the younger population due to high- velocity trauma, whereas in the elderly population it is most often due to trivial trauma. Even though the location of the fracture is similar, the differences in low $^{-}$velocity and high- velocity injuries in older compared with younger patients outweigh this similarity. High- velocity injuries are more difficult to treat and are associated with more complications when compared to low ${ }^{-}$velocity injuries. Majority of proximal femur fractures occur in elderly age groups. Nearly ninety percent occur in patients who are more than sixty five year old. Out of these, seventy five percent are woman ${ }^{[1]}$.

This has increased the demand for better and quick recovery by patient, which can be achieved to great extent by the use of newer implants like DHS, Proximal femoral nail (PFN), Interlocking nail, Gamma nail, etc. 
The risk factors for proximal femoral fractures mainly include osteoporosis, excessive alcohol consumption, high caffeine intake, physical inactivity, low body weight, poor visual acuity, previous hip fracture, smoking and malnutrition $[2,3,4$, $5,6,8]$. The etiology of the proximal femur fracture is the combination of increased bone fragility of the trochanteric area of the femur associated with decreased agility and decreased tone of the muscle in general. In addition to above factors it is also due to secondary osteoporosis due to a lack of adequate ambulation, antigravity activities, decreased anabolic hormone levels, decreased intake of and/or absorption of calcium and Vitamin D. These all contribute to increase in incidence in fracture of proximal femur ${ }^{[7]}$. While proximal femur fractures in younger individuals are as a result of high velocity injury, such as motor vehicle accidents, fall from height etc ${ }^{[10,11,12]}$.

In 1950 the proximal femur fractures were treated by immobilization and traction in bed which took very long time to unite and usually resulted in shorting of limb, mal-union and deformity due to inability of the traction to be effectively maintained with the problems of recumbency until fracture healing occurred (which usually takes more than 12 weeks). This will be followed by lengthy programme of physiotherapy. In addition, this conservative method of treatment was associated with high complication rates; like decubitus ulcers, urinary tract infection, joint contracture, pneumonia and thromboembolic complications etc. which has resulted in high mortality rate ${ }^{[9]}$.

These complications has been brought down to great extent by improved operative technique, newer antibiotic and better quality of implants which has decreased many complications. Nearly all fracture are treated by internal fixation and remains the treatment of choice with early mobilization. However, researcher are still looking for the better ways to treat these proximal femur fracture to get anatomical fixation and good outcome ${ }^{[13]}$.

Hence new implant designs such as intramedullary nails have recently changed the whole scenario in treating extra-capsular proximal femur fracture, subtrochanteric fractures even in comminuted and unstable fractures.

Proximal femoral nail (PFN), which is also a collapsible device with added rotational stability, is the latest device for the management of trochanteric fractures. This implant is a biomechanically more sound can be performed with a small incision and minimal blood loss.

The intramedullary nails have advantages in the form of more efficient and early load bearing, decrease tensile strain on the implant. This in turn decreases the risk of implant failure, less of soft tissue damage, blood loss and also saves operative time and anesthesia, resulting in decreased overall morbidity ${ }^{[20]}$. Presently with proper indication this is method of choice for treatment of proximal femoral fractures.

\section{Material \& Methods}

The present study is retrospective analysis. This study was carried out at Dhiraj Hospital, Piparia, District VADODARA from November 2014 to November 2016. The study consist of a total of 60 patients with close proximal extracapsular femur fractures treated with proximal femoral nail.

\section{Inclusion criteria}

1. Closed Fracture

2. Patient aged 18 years and above.

3. All patients with extra capsular proximal femur fractures treated with PFN
4. Boyd and Griffin type I, II, III, IV

5. Frank Seinsheimer type I, II, III, IV

\section{Exclusion Criteria}

1. Patients treated conservatively.

2. Open fracture

3. Pathological fractures

4. Patients under 18 years

\section{Data Collection}

After the patient with intertrochanteric or subtrochanteric fracture was admitted to hospital all the necessary clinical details were recorded in proforma prepared for this study. After the completion of the hospital treatment, patients were discharged and called for follow- ${ }^{-}$up at the outpatient level at regular intervals for serial clinical and radiological evaluation. The patients were followed up till fracture union and functional recovery after surgery at regular intervals and if necessary subsequent follow- up was done.

\section{Management of Patients}

As soon as the patient with suspected trochanteric or subtrochanteric fracture was seen, necessary clinical and radiological evaluation done and admitted to the ward after necessary resuscitation and splintage using skin traction. The blood, urine and $\mathrm{X}^{-}$ray investigations were done routinely on all the patients preoperatively.

\section{Preoperative measure of nail dimension}

Nail length in case of long PROXIMAL FEMORAL NAIL is determined by measuring the length from tip of greater trochanter to superior pole of patella of the normal limb. Diameter of the nail is measured by measuring the width of medullary canal at the level of isthmus on true size $\mathrm{x}$-ray. Femoral screw is measured on X-ray of opposite hip from lateral cortex to subchondral bone of femoral head.

\section{Operative Technique}

The patient is placed in supine position on a fracture table with adduction of the affected limb by $10-15^{\circ}$ and closed reduction of the fracture was done by the traction and internal rotation. The image intensifier was positioned so that anterior- $^{-}$posterior and lateral views of hip and femur could be taken. The patient is then prepared and draped as for any standard hip fracture fixation.5 $\mathrm{cm}$ longitudinal incision was taken proximal from the tip of the greater trochanter. A parallel incision was made in fascia lata, and gluteus medius was split in line with the fibers. In AP view on $\mathrm{c}^{-}$arm, the entry point is on the tip or slightly lateral to the tip of greater trochanter. In lateral view, guide wire position is confirmed in the center of the medullary cavity. Medullary canal entered with a curved bone awl; the guide wire is inserted into the medullary canal. Using a cannulated conical reamer proximal femur is reamed for a distance of about $7 \mathrm{~cm}$. After confirming satisfactory fracture reduction, an appropriate size nail as determined preoperatively is assembled to insertion handle and inserted manually. A $2.8 \mathrm{~mm}$ guide wire is inserted through the drill sleeve after a stab incision. Drilling is done over $2.8 \mathrm{~mm}$ guide wire until the drill is $8 \mathrm{~mm}$ short of the tip of the guide wire. Distal locking is usually performed with two cortical screws. Sterile dressing applied over wound and compression bandage given. Foot end elevation was given. 


\section{Postoperative Protocol}

- Post-operative check x-ray was taken.

- Intra-venous antibiotics are given to all patients for first 5 days and converted to oral antibiotics and continued till suture removal. Usually removal of suture is done on 11th post-operative day.

- Dressing is seen on 3rd, 7th and 11th post-operative days.

- Physiotherapy: Patient is taught static quadriceps exercises in the immediate postoperative period. Knee bending exercises, high sitting exercises and active quadriceps strengthening exercises are started from 3rd postoperative day, as soon as patient is comfortable and has gained adequate quadriceps strength.

- According to fixation, postoperative non weight bearing or partial weight bearing with crutch is started.

- Patient is discharged usually after suture removal.

\section{Follow up}

- After discharge patients were assessed clinically and radio logically at 4 th, $8^{\text {th }}$ and 12 th weeks, then monthly up to union of fracture and further as per requirement. In every follow up visit patients were assessed clinically for hip/thigh pain, walking ability, abnormal gait/ limp (abductor lurch, short limb gait), ,limb length discrepancy (shortening), any deformity, range of movement at hip and knee joint, muscle strength and ability to squat and sit cross-legged. Radiographs were taken to assess union and to calculate the neck shaft angle of the operated hip.

- The functional and clinical outcome is calculated on Harris hip score on every visit in terms of amount of pain, ability to walk, walking distance, muscle power, range of movement of the affected hip, participation in daily routine activities, ability to squat and sit.

\section{Results}

\section{Age and gender distribution}

In present study, age varied from 18 to 80 years. Out of all $43.4 \%$ of the patients belong to the age group of 61 to 80 years. Maximum age $85 y$ rs and minimum age 20 yrs.

In our study, we observed that out of 60 cases included in the study, 42 patients $(70 \%)$ were male and only 18 patients (30 $\%)$ were female.

Side affected:-Out of 60 patients, 34 of them had fractures on left side and 26 of them on right side.

Mode of injury:-Road traffic accident (43.3\%) is most common injury in our study. Fall while walking $(36.7 \%)$ is second most common injury in present study.

Fracture type:-All intertrochanteric fractures were classified as Boyd and Griffin classification while subtrochanteric fractures were classified as Seinsheimer classification.

Table 1: Trochanteric fractures are classified according to: Body and griffin classification

\begin{tabular}{|c|c|c|}
\hline Fracture type & No of patients & Percentage \\
\hline Boyd and griffin-1 & 3 & 5 \\
\hline Boyd and griffin-2 & 14 & 23.3 \\
\hline Boyd and griffin-3 & 5 & 8.3 \\
\hline Boyd and griffin-4 & 23 & 38.3 \\
\hline Total & 45 & \\
\hline
\end{tabular}

Table 2: Subtrochanteric fractures are classified according seinsheimer classification.

\begin{tabular}{|c|c|c|}
\hline Type & No. of patients & Percentage \\
\hline 1 & 2 & 3.3 \\
\hline $2 \mathrm{a}$ & 2 & 3.3 \\
\hline $2 \mathrm{~b}$ & 5 & 8.3 \\
\hline $2 \mathrm{c}$ & 2 & 3.3 \\
\hline $3 \mathrm{a}$ & 3 & 5 \\
\hline $3 \mathrm{~b}$ & 1 & 1.6 \\
\hline 4 & 0 & 0 \\
\hline 5 & 0 & 0 \\
\hline Total & 15 & \\
\hline
\end{tabular}

In this series, there were 45 patients $(75 \%)$ had intertrochanteric fracture while15 patients $(25 \%)$ had sub trochanteric fracture.

Reduction:-All the fractures were reduced by taking the patient on fracture table by close method except 3 case that required open reduction. There were markedly comminuted fracture which required open reduction.

Early Complication:-In present study 4 patients had superficial skin infection.

Late complications:-We had 3 case with reverse $\mathrm{Z}^{-}$phenomenon. That was managed with screw removal after 3 months. We encountered 2 cases of delayed union and 2 cases of mal- union. 3 cases had shortening which were treated with sole raise.

Follow-up duration:-In our study we had found that average follow up period was 19.03 months. Longest follow up was 36 months.

Functional results:-In our study we had 4 patient (6.6\%) experienced occasional pain and 4 patients $(6.6 \%)$ had limp while walking. In present study, $86.7 \%$ patients can squat easily. In present study, $83.3 \%$ patients can cross leg easily. In present study 2 patients $(3.3 \%)$ had $1 \mathrm{~cm}$ shorting and 1 patient $(1.6 \%)$ had $2 \mathrm{~cm}$ shorting. In our study, mean neck shaft angle was 128.6 degree. 54 patients (90\%) had neck shaft angle between 121 to 135 degrees.

Here in this study, only 3 patients $(5 \%)$ had $\mathrm{Z}$ - effect on $\mathrm{x}$ ray and 1 patient $(1.6 \%)$ had collapse. our study mean time of union was 11.7 weeks, where minimum of 10 weeks and maximum of 18 weeks.

Assessement is based on Harris Hip scoring system adopted. In this study 48 patients had excellent harris hip score $(>90)$, 6 patients had good score (80-90), 5 patients had fair score $(70-80)$ and 1 patient had poor score $(<70)$. So in present study $90 \%$ patients had Harris Hip Score value $>80$.

\section{Discussion}

Extracapsular proximal femur fractures have been recognized as a major challenge by the Orthopaedic community especially unstable. This is not solely to achieve fractures union, but for restoration of optimal function in the shortest possible time that to with minimal complications. The aim of management accordingly has drifted to achieving early mobilization, rapid rehabilitation and quick return of individuals to pre fracture stage as a functionally and psychologically independent unit.

Operative treatment in the form of internal fixation permits early mobilization and offer the best chance of functional 
recovery, hence it has become the treatment of choice for majority of fractures in the trochanteric and subtrochanteric region. Amongst the various types of implants available i.e. fixed nail plate devices, sliding nail/screw plate and intramedullary devices, the compression hip screw is most commonly used but recently techniques of closed intramedullary nailing have gained popularity with good outcome.

There are stable and unstable types of proximal femur fractures, but in case of unstable variety with loss of posteromedial cortex, there is impaction with shortening of the neck of femur thereby reduction of the lever arm of the hip abductors. Most of the body weight is transferred by the calcar and a plate supporting a nail or screw which would be at a distance lateral to this weight bearing line, produces considerable tension on the implant. Proximal femoral nail is closer to the calcar, subjected to less tension and is more stable and better fixation ${ }^{[21]}$.

Proximal femoral nail insertion is accomplished closed through a smaller skin incision preserving the fracture hematoma, which is an essential element in fracture healing. The decrease in surgical trauma certainly reduces intraoperative blood loss, infection and wound complications, allowing significantly earlier rehabilitation and a shorter hospital stay ${ }^{[22]}$.

PFN is a novel, modern intramedullary implant based on experience with the gamma nail. The currently used gamma nail as an intramedullary device also has a high learning curve with technical and mechanical failure rates of about $10 \%$ (collapse of the fracture area, cut out of the implant, fracture of the femur shaft). The gamma nail is susceptible to fail at its weakest point, the lag screw implant interface. The arbeitsgemeinschaft fur osteosynthesefragen in 1996 therefore, developed the PFN to reduce the risk of implant related complications. In addition to the $8 \mathrm{~mm}$ load bearing femoral neck screw, the PFN has a $6.5 \mathrm{~mm}$ antirotation screw to increase the rotational stability of the neck fragment. An anatomic $6^{\circ}$ neck valgus bend in the coronal plane, a narrower distal diameter and distal flexibility of the nail eliminates the need for routine reaming of the femoral shaft and also minimizes stress concentration and tension in the femoral shaft. Jiang et al. ${ }^{[23]}$ recommended that the lag screw of PFN should be placed in the lower part of the femoral neck close to the femoral calcar, with screw tip reaching the subchondral bone $5-10 \mathrm{~mm}$ below the articular cartilage in anteroposterior view. In lateral view, it should be placed in the center of the femoral neck. There, the lag screw will be definitely placed in the area of best bone quality. PFN has all advantages of an intramedullary device, such as decreasing the moment arm, can be inserted by closed technique, which retains the fracture hematoma an important consideration in fracture healing, decrease blood loss, infection, minimizes soft tissue dissection, and wound complications.

In this study an attempt was made to survey, evaluate, document and quantify our result in the management of such patients by using proximal femoral nail (PFN) and I had used Harris hip score for the assement of the results.

We had taken 60 cases of extracapsular proximal femur fractures during the period from November 2014 to November 2016 at Dhiraj General Hospital, Piparia, Vadodara. The minimum follow up period was six months. I evaluated results and compared them with those obtained from various published studies.

In my study, proximal femur fractures were more common after 5 th decade with a mean age group of 56.4 years where as in the studies of Franco et al. (2008) ${ }^{[24]}$, Shan-Wei Yang et al.(2012) ${ }^{[25]}$ and Bhakat et al.(2013) ${ }^{[26]}$ who had an mean age of 79.3 years, 53 years and 67.8 years respectively.

The sex ratio as per this study was male: female $=2.3: 1$. Out of 60 patients, there were 42 male $(70 \%)$ patients and 18 female (30\%) patients. While in Franco et al. (2008) ${ }^{[24]}$ and Bhakat et al. (2013) ${ }^{[26]}$ study, more patient were female 81 out of 111 $(73 \%)$, and 17 out of $30(60 \%)$ respectively. In our study increased incidence in male as compare to female is due to their involvement in riding vehicles, heavy agriculture, labour and more outdoor activities. Most common mode of injury in my study was high energy trauma in $63.3 \%$ patients which include road traffic accidents (43\%) and fall from height (20\%). While $36.7 \%$ patients had low energy trauma due to fall while walking which was most commonly seen in elderly patients. These were also attributed to the high incidence of osteoporosis specially in elderly patients. In study of ShanWei Yang et al. (2012) ${ }^{[25]} 60 \%$ patients had high velocity injury.

In our study, we had used Boyd and griffin classification for intertrochanteric fractures and Seinsheimer classification for Subtrochanteric fractures. Out of 60 patients there were 45 patient $(75 \%)$ had intertrochanteric fracture while 15 patient $(25 \%)$ had sub-trochanteric fracture.

All cases were operated on fracture table. In 57 patients we achieved close reduction but in 3 patients open reduction was required which were Boyd and Griffin type 4 and Seinsheimer type 3 variety and we were not able to achieve anatomical reduction by closed reduction.

In our study, we had not used any prophylactic antithrombotic agents and there were no cases of deep vein thrombosis, pulmonary embolism, and wound healing problems. While in study of S.K Venkatesh (2015) ${ }^{[27]}$, there was 2 case of deep vein thrombosis. In this series, this is in favour of early mobilization after surgery with non-weight bearing.

There were no complications in form of fracture at tip of trochanter which occurs because of improper entry and can be avoided by making proper entry point.Malrotation which usually occurs during insertion of large sized hip screw was avoided by proper reduction and stabilizing the proximal fragment with derotation hip screw.

In all patients, non-weight bearing mobilization was started on post-operative day one and in $80 \%$ patient full weight bearing started on day of discharge which is usually 12th postoperative day.

In our study, the superficial infection has occurred in 4 patients $(6.6 \%)$. Both of these cases were satisfactorily managed by parenteral antibiotic and regular dressings. While in Franco study (2008) ${ }^{[24]}$, Shan-Wei Yang study (2012) ${ }^{[25]}$ and Bhakat and Ranadeb study (2013) ${ }^{[26]}$, there were no infection. This is probably due to poor hygiene condition of our patients. There is no case of deep infection in my study.

In our study, average follow up period was 19.03 months and longes follow up was 36 months and minimum follow up was 6 months.

In this study, 4 patients had occasional pain on final follow up and because of that they had limp while walking. Pain was relieved on taking analgesics. Out of 60 patients, 3 patients $(10 \%)$ had difficulty on cross leg sitting and squatting. At final evaluation, implant status was evaluated radio logically once the fracture was united and checked for any kind of implant related complication or failure. We found 3 cases $(5 \%)$ with ' $Z$ ' effect where there was intrusion of the proximal hip screw into the joint and back out of the lag screw. The Z-effect involves the lateral migration of the 
inferior screw, varus collapse of the fracture and perforation of the femoral head by the superior screw. The first account of the Z-effect has been attributed to Werner-Tutshcku et al. 91, who reported a series of 70 cases of fractures treated using PFN. These authors also advised that fixation of the fracture at neck shaft angle of $<125^{\circ}$ is a predisposing factor for the Zeffect.

Although the cause of this complication has been explained by varus collapse of the fracture and the lack of medial cortical support ${ }^{[28]}$. Here in this series, there was varus seen in case of Z-effect. In these cases, screws were changed later on.W. M.Gadegone and Y. S. Salphale ${ }^{[29]}$ found that in three patients out of hundred cases studied with PFN, ' $Z$ effect' was noted with the migration of hip pins into the joint.In my study, limb length was measured regularly and final limb length was measured when the fracture has radio logically united.

The limb length was measured and compared to the normal limb. Out of 60 patients, 57 patients $(95 \%)$ had no limb length discrepancy and 3 patients $(5 \%)$ had limb shortening which are $1 \mathrm{~cm}, 1 \mathrm{~cm}$ and $2 \mathrm{cms}$ respectively. In these 3 patients shortening are due to a combination of fracture communition and collapse. One patient had shortening of $2 \mathrm{cms}$ which had coxavara on $\mathrm{x}$ ray and in this patient shortening were compensated by shoe raise elevation. In study of Bhakat and Ranadeb (2013) et al. ${ }^{[26]}$, there was shortening of $0.5 \mathrm{~cm}$.

I have used criteria for union as absence of pain at fracture site clinically and presence of bridging callus at fracture site radio logically. The average time of fracture union in my study was 11.7 weeks, ranging from 10 weeks to 22 weeks. In study of S.K.Venkatesh (2015) et al. ${ }^{[27]}$ average time of union was 8 weeks.

In present series, depend upon Harris hip score, the result shows excellent results in the 21 patients $(70 \%)$, good results in 6 patients $(20 \%)$, fair results in 2 patients $(6.7 \%)$ and poor in 1 patient $(3.3 \%)$. This mean Harris Hip Score in present series was $90 \%$. While S.K.Venkatesh (2015) et al. [27] excellent result in $66.2 \%$, good results in $28.2 \%$ and fair result in $5.6 \%$.

In this study, 45 cases were intertrochanteric type according to Boyd and Griffin classification. Among them 39 patients has excellent result, 3 patients had good result (type 4) and 3 had fair result (type 4).

While there were 15 cases of subtrochenteric type according to seinsheimer classification. Among them 9 patients had excellent result, 3 had good result( 1 case of type $3 \mathrm{a}$ and 2 case of type $2 b), 2$ had fair result(type $2 c$ and $3 a$ ) and 1 had poor result (type $3 b$ ).

Proximal Femoral Nail is based on sound bio-mechanically principles. PFN helps in least blood loss, early mobilization and weight bearing even in unstable fractures, thus providing good functional recovery and early fracture union with excellent results. I think this is the best treatment available for proximal femur fracture in present scenario.

Table 3

\begin{tabular}{|c|c|c|c|c|}
\hline & Excellent & Good & fair & Poor \\
\hline Intertrochenteric & 39 & 03 & 03 & 00 \\
\hline subtrochenteric & 09 & 03 & 02 & 01 \\
\hline
\end{tabular}

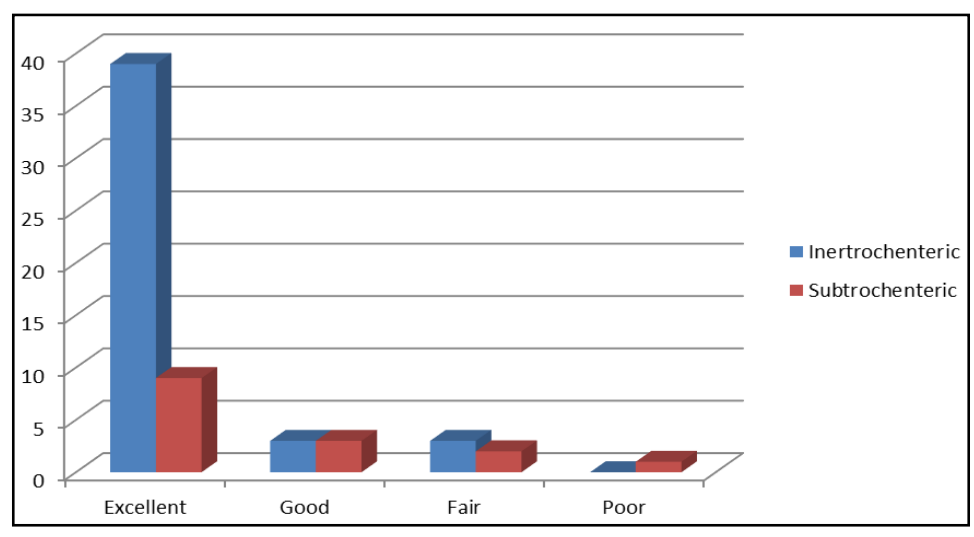

Fig 1

\section{Conclusion}

We have studied 30 cases of extracapsular proximal femur fractures treated with proximal femur nail. The clinical results and functional outcomes were compared with other study in order to find out the best outcome for such fractures which is calculated according to Harris Hip scoring system.

PFN has the advantage of collapse at the fracture site and is biomechanically sound as it is done by closed technique, fracture opened only when closed reduction could not be achieved, and it is an intramedullary device. Another advantage of this device is it prevents excess collapse at fracture site thus maintaining neck length. Osteosynthesis with the PFN offers the advantages of high rotational stability of the head- neck fragment. The two neck screws should be placed in the center of neck and head, the proximal one act as derotation screw and the distal one as collapsing screw. Postoperatively early mobilization can be begun as the fixation is rigid and because of the implant design.

Hence, it was concluded that, though the learning curve of this procedure is steep, with proper patient selection, good instrumentation, image intensifier and surgical technique, PFN remains the implant of choice in the management of proximal third fracture of the femur.

\section{Clinical pictures}
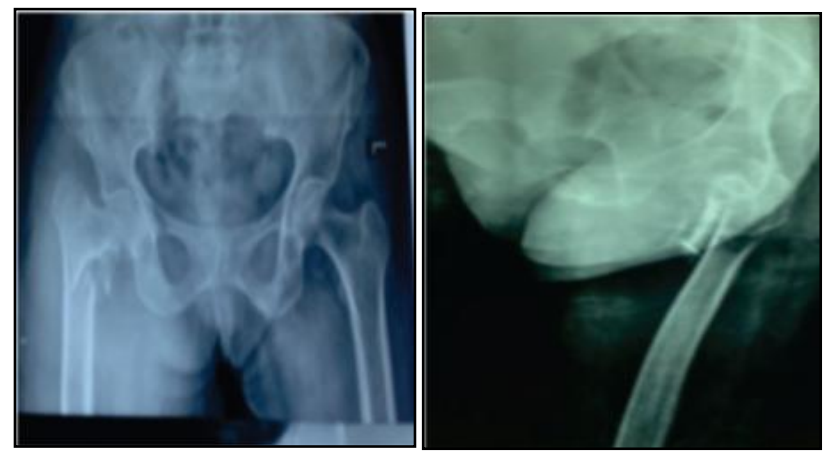

Fig 2: Pre-op X-ray 

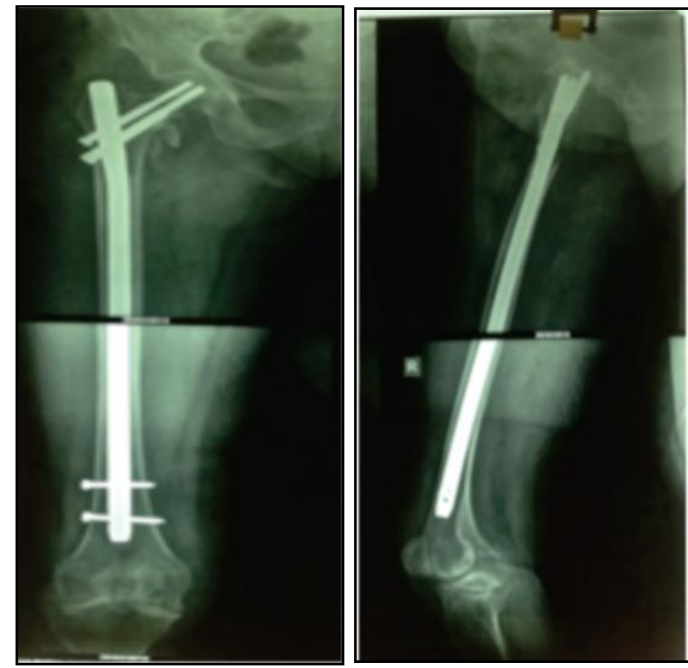

Fig 3: Post - op x ray

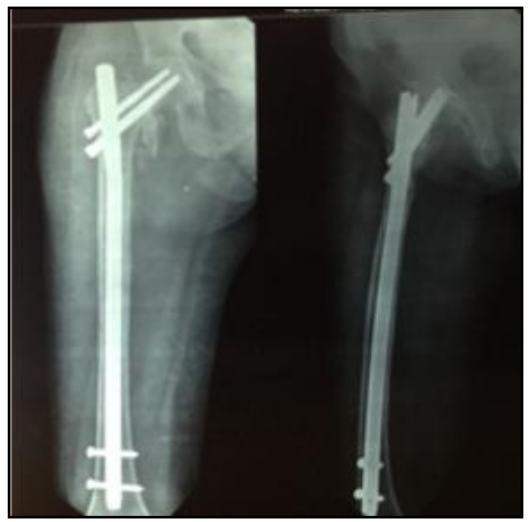

Fig 4: Final follow up x ray

\section{References}

1. Lorich, Dean GMD et al. Osteoporotic Pertrochanteric hip fractures management and current controversies JBJS Am. 2004; 86:398-410.

2. Guyton JL. Fractures of hip, acetabulum, and pelvis. In: Canale ST, editor. Campbell's operative orthopaedics. 9th ed. St. Louis: Mosby, 1998, 2181-27.

3. Hernandez-Avila M, Colditz GA, Stampfer MJ, et al. Caffeine, moderate alcohol intake, and risk of fractures of the hip and forearm in middle-aged women. Am J Clin Nutr. 1991; 54:157-63.

4. Paganini-Hill A, Chao A, Ross RK, Henderson BE. Exercise and other factors in the prevention of hip fracture: the Leisure World study. Epidemiology. 1991; 2:16-25.

5. Farmer ME, Harris $\mathrm{T}$, Madans $\mathrm{JH}$, et al. Anthropometricindicators and hip fracture. The NHANES I epidemiologic follow-up study. J Am Geriatr Soc. 1989; 37:9-16.

6. Farmer ME, Harris T, Madans JH, et al. Anthropometric indicators and hip fracture. The NHANES I epidemiologic follow-up study. J Am Geriatr Soc. 1989; 37:9-16.

7. http://li593-83.members.linode.com/node/1220623

8. McGrory BJ. Stinchfield resisted hip flexion test. Hosp Physician. 1999; 35(9):41-2.

9. Parker $\mathrm{MH}$. Conservative versus operative treatment for extracasular hip fracture. Cocharane Database sys rev. 2000; (2).

10. Bedi A, Tonale T. Subtrochanteric femur fracture. Orthop Clin N Am. 2004; 35(4).
11. Fielding JW, Magliato HJ. Subtrochanteric fracture. Surg Gynecol Obstet, 1966, 122.

12. Kinast C, Bolhofner BR, Mast JW, et al. Subtrochanteric femur fracture, results of treatment with the 95 condylar blade plate. Clin orthop, 1989, 28.

13. Ricci, William MMD. New implants for the treatment of intertrochantric femur fractures. Techniques in orthopedics. 2004; 19(3):143-152.

14. Doppelt SH. The sliding compression screw. Today's best answer for stabilization of intertrochanteric hip fractures. Orthopclin North A.II, 1980, 507-523.

15. Simpson AHRW, Varty K, Dodd CAF: Sliding hip screws: Modes of failure.Injury. 1989; 20:227-231.

16. Wolfgan Gl, Blyart MH, O'Neil JP. Treatment of intertrochanteric fracture of the femur using sliding screw plate fixation. Clin Orthop. 1982; 163:148-158.

17. Davis TRC, sher JL Horsman S, et.al. Intertrochanteric femoral fractures: Mechanical failure after internal fixation. JBJS Br. 1990; 72:26-31.

18. Kaufer H. Mechanics of the treatment of hip injuries. Clin. Orthop. 1980; 146:53-61.

19. Rosenblum Sf, Zuckerman JD, Kummer FJ et.al. A biochemical evaluation of the Gamma nail. JBJS Br. 1992; 74:353-357.

20. Kemmeth J Koval, Joseph D. Zuckerman: Rockwood and Green's fracture in Adults, chapter 39, 5th edition- edited by Robert W.Buchlog and James D. Heckman, J.B.Lipincott Company. 2001; 2:1635-1663.

21. Halder Sc. The gamma nail for peritrochanteric fractures. JBJS (Br). 1992; 74:340-4.

22. Christian Boldin, Franz J Seibert, Florian et al. The proximal femoral nail (PFN) - a minimal invasive treatment of unstable proximal femoral fractures. A prospective study of 55 patients with a follow up of 15 months. Acta Orthop Scand. 2003; 74(1):53-58.

23. Jiang LS, Shen L, Dai LY. Intramedullary fixation of subtrochanteric fractures with long proximal femoral nail or long gamma nail:Technical notes and preliminary results. Ann Acad Med Singapore. 2007; 36:821-6.

24. Franco Lavini, L Renzi-Brivio, R Aulisa, F Cherubino, PL Di Seglio, N Galante, et al. The treatment of stable and unstable proximal femoral fractures with a new trochanteric nail: results of a multicentre study with the VeronailStrat Traum Limb Recon. 2008; 3:15-22.

25. Fu-Ting Huang, Md Kai-Cheng Lin, Md Shan-Wei Yang, Md Jenn-Huei Renn, Md Comparative Study of the Proximal Femoral Nail Antirotation Versus the Reconstruction Nail in the Treatment of Comminuted Proximal Femoral Fracture: doi: 10.3928/0147744720111122-02

26. Ujjal Bhakat, Ranadeb Bandyopadhayay Comparitive Study between Proximal Femoral Nailing and Dynamic Hip Screw in Intertrochanteric Fracture of Femur: Open Journal of Orthopedics. 2013; 3:291-295.

27. SK Venkatesh Gupta, Veera Shekar Valisetti Comparative study between dynamic hip screw vs proximal femoral nailing in inter-trochanteric fractures of the femur in adults: IJOS. 2015; 1(1):07-11.

28. Strauss EJ, Kummer FJ, Koval KJ, Egol KA. The Zeffect phenomenon defined: a laboratory study. J Orthop Res. 2007; 25:1568-1573.

29. WM Gadegone, YS Salphale. Proximal femoral nail - an analysis of 100 cases of proximal femoral fractures with an average follow up of 1 year. Int Orthop. 2007; 31(3):403-408. 